\title{
CARACTERIZAÇÃO DE LATOSSOLO AMARELO SOB CULTIVO CONTÍNUO DE CANA-DE-AÇÚCAR NO ESTADO DE ALAGOAS: ATRIBUTOS MORFOLÓGICOS E FÍSICOS ${ }^{(1)}$
}

\author{
A. J . N. da $\operatorname{SILVA}^{(2)} \&$ M. R. RIBEIRO ${ }^{(3)}$
}

\begin{abstract}
RESUMO
Foram estudados os efeitos do cultivo contínuo da cana-de-açúcar nas principais propriedades físicas e morfológicas de um latossolo amarelo argiloso da região dos tabuleiros costei ros do estado de Alagoas. Quatro talhões foram selecionados na Usina Caeté, município de São Miguel dos Campos (AL), em janeiro de 1995; um com vegetação nativa (Tn) e os demais cultivados por períodos de dois (T2), dezoito (T18) e vinte e cinco (T25) anos. Os solos foram caracterizados morfologicamente e, nas amostras de cada horizonte, foram determinados granulometria, grau de floculação, umidade a -0,034 MPa e -1,52 MPa, densidade do solo e das partículas, macroporosidade e porosidade total. Foram coletadas amostras indeformadas dos horizontes A, AB e BA de cada perfil para determinação de condutividade hidráulica saturada, microporosidade e densidade do solo. Os resultados mostraram que o uso agrícola dos solos causou mudanças na morfologia do horizonte superficial com o desenvolvimento de um horizonte Ap, apresentando transição abrupta para o horizonte $A B$ subjacente e perda de estrutura. Em relação às propriedades físicas, os resultados indicaram que o cultivo contínuo causou diminuição no conteúdo de argila, nos horizontes superficiais, e um aumento significativo, nos horizontes subsuperficiais. Após evidente impacto negativo nas propriedades físicas com o primeiro plantio da cana-de-açúcar, o manejo adotado promoveu novo equilíbrio, com recuperação parcial da porosidade total e sensível aumento no conteúdo de água disponível. Por outro lado, o uso agrícola promoveu significativa redução na condutividade hidráulica saturada do horizonte superficial, quando comparado com o solo sob vegetação nativa.
\end{abstract}

Termos de indexação: latossolo amarelo, propriedades físicas, compactação, cultivo contínuo, cana-de-açúcar.

\footnotetext{
(1) Parte da dissertação de Mestrado do primeiro autor, apresentada à Universidade Federal Rural de Pernambuco, para obtenção do grau de Mestre em Agronomia - Ciência do Solo. Trabalho apresentado no XXVI Congresso Brasileiro de Ciência do Solo, Rio de J aneiro (RJ ), 20 a 26 de julho de 1997. Recebido para publicação em setembro de 1996 e aprovado em outubro de 1997.

(2) Engenheiro-agrônomo, aluno do curso de Mestrado em Agronomia-Ciência do Solo da UFRPE. Rua Bom J ardim, no 125, BL 1, apto 303, J ardim Brasil I, CEP 53230-520 Olinda (PE).

(3) Professor Adjunto do Departamento de Agronomia da Universidade Federal Rural de Pernambuco. Rua Dom Manuel de Medeiros, s/n, CEP 52171-900 Recife (PE). Bolsista do CNPq.
} 


\begin{abstract}
SUMMARY: CARACTERIZATION OF XANTHIC FERRALSOL UNDER CONTINUOUS SUGARCANE CROPPING IN ALAGOAS STATE, BRAZIL: MORPHOLOGICAL AND PHYSICAL ATTRIBUTES
\end{abstract}

\begin{abstract}
Theeffects of continuous sugarcanecropping on thephysi cal and morphological properties of a clayey xanthic ferralsol werestudied in theregion of the Low Coastal Tablelands of Alagoas State, Brazil. Four sites were selected at Caeté mill, São Migued dos Campos, State of Alagoas, Brazil, involving a native forest (Tn), and sugarcane fields, cultivated for periods of two (T2), eighteen (T18) and twenty-five (T25) years. The soils were morphologically characterized, and samples weretaken from each horizon to determi neparticlesizedistribution, degreof floculation, moisture at - $0.034 \mathrm{MPa}$ and - $1.52 \mathrm{MPa}$, soil bulk and particle densities, macroporosity and calculated total porosity. Undisturbed samples of the A, AB and BA horizons of each profile werecollected to determi nemicroporosity, saturated hydraul ic conductivity and soil bulk density. The results showed that the agricultural use promoted morphological changes with the development of an Ap horizon showing abrupt transition to the underlying AB horizon and lack of structure In relation to physical properties, the results indicated that the cultivation decreased the clay content of the surface horizon and increased significantly that of the subsurfacehorizon. After an evident negativeimpact on physical properties after the first crop, the management practices promoted a new equilibrium, with increases in total porosity and water availability. On the other hand, the agricultural use resulted in a significant reduction of the saturated hydraulic conductivity in the surface horizon, when compared to the virgin forest site
\end{abstract}

I ndex terms: Clayey xanthic ferral sol , physical properties, soil compaction, continuous-cropping, sugarcane

\section{INTRODUÇÃO}

Nos últimos vinte anos, as áreas plantadas com a cultura da cana-de-açúcar têm-se expandido, consideravel mente, no Brasil, que é hoje o maior produtor mundial dessa cultura, com uma área de cultivo cal culada em 4,2 milhões dehectares, em 1994, e uma produção de 240 milhões de tonel adas de cana, 9,5 milhões de toneladas de açúcar e 12 bilhões de litros de álcool (Orlando Filho et al., 1995). Tal desempenho reflete um vasto programa de produção de álcool a partir da cana-de-açúcar, que vem sendo desenvolvido no País desde 1974.

O cultivo contínuo e o preparo do sol o para o plantio provocam diversas alterações nas suas propriedades físicas, resultando na degradação da estrutura do solo. A vel ocidade dessa degradação pode ser acel erada pelo uso intensivo do solo, sendo comum a presença de camada compactada em subsuperfície ou ocorrência decrostas superficiais (Correia, 1985a; Cerri et al, 1991; Meek et al., 1992; Corsini, 1993; Goves et al., 1994).

As modificações importantes ocorridas em solos que sofreram compactação dizem respeito ao aumento da resistência mecânica à penetração radicular, à redução da aeração (M cAfee 1989; Perez Filho et al., 1993), a alterações na disponibilidade efluxo deágua, calor e nutrientes. Em determi nado tempo e local, um desses fatores pode tornar-se importante para o desenvolvimento das plantas, dependendo do tipo de solo, condição dimática, espéciee estádio de desenvol vimento da planta (Camargo, 1983; Farias et al., 1985).

Vários trabal hos mostram queo manejo inadequado e o tempo de cultivo contínuo afetam a porosidade do sol o e, em particular, a sua macroporosidade. Romero de Carvalho et al. (1991) e Silva \& Ribeiro (1992), estudando o efeito de vários anos de cultivo convencional com cana-de-açúcar, verificaram que, nos solos com maior tempo de cultivo, a porosidade diminuía e, com maior evidência, a macroporosidade. A condutividade hidráulica saturada também sofre alterações expressivas com o cultivo contínuo, poi s está intimamente associada com o volume dos poros, especial mente os chamados macroporos ou por os nãocapilares, responsáveis pela drenagem, percolação e aeração do solo (Correia, 1985b).

Os solos utilizados no cultivo da cana-de-açúcar no Brasil vão desde areias quartzosas a latossolos, sendo estes últimos, juntamente com os podzólicos, os mais representativos. No Estado de Alagoas, grande parte das áreas cultivadas com a cana-de-açúcar estão incluídas em solos de tabuleiros costeiros. Considerando a sua extensão, a incorporação dos tabuleiros ao sistema produtivo resultou em grandes benefícios à economia regional, principalmente na produção da cana-de-açúcar.

As deficiências naturais existentes nos solos de tabuleiros foram contornadas pelo desenvolvimento de práticas e tecnol ogias aparentemente adequadas. No entanto, nos últimos anos, verificou-se que as técnicas de cultivo empregadas pelos agricultores e empresas do ramo poderiam provocar danos às propriedades desses sol os, que mostram forte coesão natural em subsuperfície, o que pode favorecer a formação de camadas compactadas, com reflexos na porosidade total, macroporosidade, estrutura e fluxo de água.

A freqüência com que se têm detectado níveis altos decompactaçãoem sol os cultivados com cana-de-açúcar, 
bem como outros tipos de degradação, requer o desenvolvimento de pesquisas para entender os mecanismos que regem o processo. O objetivo deste trabalho foi quantificar as modificações de algumas propriedades físicas e morfológicas de um latossolo amarelo argiloso cultivado com cana-de-açúcar.

\section{MATERIAL E MÉTODOS}

O trabalho foi desenvolvido em áreas localizadas na Usina Caeté, no município de São Miguel dos Campos, microrregião homogênea no 119 do litoral de Alagoa (J acomine, 1975).

Com base em levantamento pedológico detalhado feito na usina Caeté, em 1993, foram selecionados quatro perfis de latossol o amarelo coeso $A$ moderado textura argilosa, representativos de áreas com vegetação nativa e cultivadas com cana-de-açúcar por diferentes períodos de tempo.

As parcelas, com diferentes tempos de cultivo, foram comparadas entre si e em relação a uma testemunha absoluta, representada por solo em condições naturais (vegetação nativa). Os perfis receberam as seguintes identificações: Tn-solo em condição natural (vegetação nativa); T2-solo com 2 anos de cultivo; T18-solo com 18 anos de cultivo, e T25-solo com 25 anos de cultivo. Todos os perfis situam-se numa mesma posição topográfica de topo plano de baixo platô costeiro.

Em condições naturais, o latossolo amarelo se caracteriza por uma seqüência de horizontes Oo, $A$, $A B, B A, B w_{1}$ e Bw $w_{2}$, com transi ções graduais e planas, nos horizontes $A$ e $A B$, e difusas e planas, a partir do horizonteBA. Caracteristicamente, apresenta um fino manto de restos vegetais pouco decompostos, que constitui a camada Oo, a qual se seque um horizonte A, de coloração bruno-acinzentada muito escura, quando úmido, textura franco argilo arenosa e estrutura forte pequena a média granular. $O$ horizonte $A B$ revela coloração bruno-escura, quando úmido, textura franco argilo-arenosa e estrutura moderada pequena a média granular e fraca pequena a média blocos subangulares. O horizonte BA apresenta coloração bruno-amarelada, textura argila arenosa, estrutura maciça coesa e consistência muito dura e firme. O horizonte Bw, subdividido nos horizontes Bw eBw $w_{2}$, apresenta col oração bruno-amarelada, no Bw $w_{1}$ eamarelo-brunada com mosqueado vermelho, no Bw $\mathrm{B}_{2}$; a textura é argilosa e a estrutura é fraca pequena a média bl ocos subangulares, com aspecto maciço poroso no $\mathrm{Bw}_{2}$. A descrição morfológica dos quatro perfis estudados baseou-se em Lemos \& Santos (1984).

Nas áreas cultivadas, foi adotado o seguinte manejo. No primeiro plantio e a cada 6 anos, quando da renovação, foram feitas duas gradagens pesadas e abertura dos sulcos com sulcador. Também foi feita, nessa ocasião, uma adubação N-P-K, de acordo com análise do solo. Anualmente, foram administradas adubações de cobertura, feitas com base na análise do solo, equetem dado, em média, cerca de $495 \mathrm{~kg} \mathrm{ha}^{-1}$ da fórmula 16-00-24, além de 1-2 capinas e aplicação de herbicidas. Também foram aplicados cerca de $400 \mathrm{~m}^{3}$ ha-1 de vinhaça pelo método de aspersão.

Após a descrição morfológica dos perfis, procedeuse à col eta das amostras em todos os horizontes. Para determinar granulometria do solo, as amostras foram pré-tratadas, secas ao ar, destor roadas e passadas em peneiras com abertura de $2 \mathrm{~mm}$ para obtenção da TFSA (Terra Fina Seca ao Ar). Para relacionar os resultados em TFSE (Terra Fina Seca em Estufa), usou-se um fator de cor reção para expressar a relação entre a massa de TFSA e TFSE. Também foram coletadas amostras indeformadas para determinações de densidade do solo, porosidade e condutividade hidráulica saturada.

As análises físicas foram feitas no laboratório de Física do Solo da Universidade Federal Rural de Pernambuco, segundo métodos descritos no Manual de Métodos de Análise de Solo (EMBRAPA, 1979).

Os parâmetros determinados foram: densidade do solo com anel volumétrico; densidade das partículas com balãovol umétrico; microporosidade com amostras indeformadas, utilizando o funil de Buchner; porosidade total, segundo Vomocil (1965); macroporosidade, pela expressão proposta por Grohmann (1960); granulometria, pelo método da pipeta; argila dispersa em água; capacidade de campo e ponto de murcha permanente, conforme Richards (1954); água disponível e condutividade hidráulica saturada, em amostras indeformadas, através de permeâmetro, descrito por Lima (1986), e conforme a equação de Darcy-Williams, descrita por Klute\& Dirksen (1986).

\section{Tratamento Estatístico}

Estudaram-se as variações das propriedades do sol o com o tempo de cultivo e com a profundidade por meio de um del ineamento inteiramente casualizado, em esquema fatorial $4 \times 3 \times 3$, segundo Silva \& Silva (1982), correspondente a quatro diferentes tempos de cultivo, três profundidades e três repetições. Os tratamentos de interesse estudados foram determinados na elaboração do experimento e considerados como fatores fixos. Quando o tempo de cultivo e/ou profundidade e a interação entre eles mostraram-se significativos noteste "F ", a comparação das médias foi feita pelo teste de Tukey ao nível de $5 \%$ de probabilidade, para cada caso, usando-se o software SANEST (Zonta \& Machado, 1980).

\section{RESULTADOS E DISCUSSÃO}

\section{Atributos Morfológicos}

Todos os perfis foram classificados como latossolo amarel o álico coeso A moderado textura argilosa fase floresta subperenifólia relevo plano e apresentaram seqüência dehorizontes $A, A B, B A, B w_{1}$ eBw $_{2}$. O perfil sob floresta nativa (Tn) mostra um horizonteorgânico 
(Oo) de três centímetros de espessura, formado por detritos vegetais pouco decompostos. Dadas as operações necessárias ao cultivo da cana-de-açúcar, o horizonte Oo nativo da mata foi queimado e/ou incorporado ao horizonte A subjacente, ajudando na formação do horizonte Ap nos perfis cultivados. Os horizontes superficiais e subsuperficiais não apresentaram variações expressivas com relação às suas cores e espessuras.

As princi pais modi ficações morfol ógicas devidas ao cultivo contínuo dos sol os estudados foram observadas com relação à transição e estrutura dos horizontes superficiais. A transição passou de gradual, no horizonte A do perfil sob mata nativa, para clara ou abrupta, no horizonte Ap dos perfis cultivados. Com relação à estrutura, o horizonte superficial do perfil Tn sob mata nativa apresentou uma estrutura forte pequena a média granular, enquanto o Ap do perfil T2 apresentou estrutura fraca pequena a média granular e blocos subangulares e maciça moderadamente coesa, evidenciando o efeito do impacto imediato do desmatamento e cultivo no grau de estrutura do solo. Observa-se, porém, que, através dos anos (perfis T18 eT25), o cultivo proporcionou ligeira mel hora no grau de estrutura em rel ação ao perfil T2, passando para fraca a moderada pequena a média granular e blocos subangulares, o que pode ser atribuído, provavelmente, ao sistema radical fasciculado da cana-de-açúcar e ao seu caráter semi perene, com movimentação do solo a cada quatro ou seis anos.

O horizonte $A B$ mostrou tendência de degradação do grau e tipo da estrutura com o cultivo, apresentando estrutura fraca a moderada pequena a média blocos subangulares e granular no perfil sob vegetação nativa (Tn) emaciça moderadamente coesa (perfil T2) ou fraca pequena a média blocos subangulares (perfis T18 eT25) nos sol os cultivados, refletindo, também, o efeito do cultivo e a conseqüente compactação. O aspecto coeso é observado nos quatro perfis estudados a partir dos 35 centímetros. Nos perfis cultivados, esse fenômeno ocorre desde o horizonteAp, que apresenta, nas entrelinhas, estrutura com aspecto maciço moderadamente coeso, em virtude do cultivo. Entretanto, a coesão subsuperficial do horizonte BA, que ocorre também no perfil sob vegetação nativa, indica adensamento de origem genética, como comentam Panoso (1976) e Silva \& Ribeiro (1992).

Em todos os perfis, os horizontes superficiais apresentam-se ligeiramente duros ou duros efriáveis a muito friáveis, o horizonte $A B$ possui, consistência dura a muito dura e friável, e os horizontes BA mostram-se muito duros e firmes em todos os perfis. Os horizontes subsuperficiais $\left(\mathrm{Bw}_{1}\right.$ e $\left.\mathrm{Bw}_{2}\right)$ revelam consistência dura a muito dura, quando secos, e friável, quando úmidos, em todos os perfis.

\section{Atributos Físicos}

Granulometria: 0 quadro 1 mostra os resultados da granulometria nos quatro perfis estudados. Com relação à profundidade, os conteúdos de argila aumentaram, significativamente, em todos os perfis, enquanto os teores de silte aumentaram apenas no perfil Tn; por sua vez, os teores de areia grossa decresceram, significativamente, nos quatro perfis estudados, enquanto a areia fina diminuiu apenas nos perfis T18 eT25.

Com relação ao tempo de cultivo, também foram observadas mudanças expressivas nos teores das frações granulométricas. O conteúdo de argila caiu, significativamente, após 18 e 25 anos de cultivo contínuo, nos horizontes superficiais, eaumentou, nos horizontes $A B$ e BA. A redução gradativa do teor de argila, com o tempo de cultivo no horizonte superficial, e o seu aumento com a profundidade resultaram na el evação da relação textural. Esse acréscimo pode ter si do influenciado pel o cultivo, que favorece a eluviação de argila do horizonte superficial, tendo em vista que os perfis estudados ocupam a mesma posição topográfica de topo plano de tabuleiro.

O aumento do conteúdo de argila, considerando a profundidade nos quatro perfis, e o aumento com o tempo de cultivo, nos horizontes AB eBA, favoreceram o aumento da microporosidade e retenção de água do solo, com conseqüente redução da condutividade hidráulica, como será discutido adiante.

Densidade do solo e das partículas: 0 quadro 2 mostra as variações da densidade do solo, considerando o tempo de cultivo e a profundidade. Observa-se aumento significativo nos valores da densidade do solo nos horizontes $A$ e AB, aos 2 anos de cultivo (perfil T2), em relação ao sol o sob vegetação nativa. Tais val ores decresceram, significativamente, até atingir, após 25 anos de cultivo, valores semel hantes aos verificados no sol o sob mata virgem. No horizonte BA, não houve mudanças com o tempo de cultivo, visto que a densidade do solo já se apresentava alta no solo virgem, comprovando a origem pedogenética da coesão desse horizonte. Tais resultados evidenciam a ocorrência degrandeimpacto com a remoção mecânica da vegetação natural e primeiro plantio da cana-de-açúcar, aumentando, drasticamente, a densidade do sol o aos dois anos, nos horizontes superficiais ( $A$ e AB). Ao longo dos anos, 0 sistema radicular fasciculado da cultura e o seu caráter semi perene, com movimentação do solo apenas a cada seis anos, favoreceram a recuperação da densidade do solo para valores próximos à situação do solo virgem. Nesses solos, percebe-se compactação nos horizontes $A p$ e $A B$, aos dois anos de cultivo, causada por meios mecânicos, com destruição dos agregados do solo e posterior aproximação das partículas. No entanto essa compactação foi recuperada, parcialmente, aos 18 e 25 anos de cultivo.

Porosidadetotal, macro e microporoside: O quadro 2 mostra as percentagens da por osidade total, macro emicroporosidade nos horizontes estudados. Observase variação no volume de poros do solo, com clara predominância de microporos, exceto no horizonte superficial do perfil Tn, cujos valores de macro e microporos são praticamente iguais. No horizonte superficial do perfil sob mata nativa (Tn), as condições naturais favoreceram o desenvolvimento da macroporosidade, enquanto o valor obtido no perfil 
Quadro 1. Granulometria do solo nos quatro perfis estudados

\begin{tabular}{|c|c|c|c|c|c|}
\hline \multicolumn{2}{|c|}{ Horizonte } & \multicolumn{4}{|c|}{ Frações granulométricas } \\
\hline Símbolo & Espessura & Areia grossa & Areia fina & Argila & Silte \\
\hline \multicolumn{6}{|c|}{$\mathrm{m}$} \\
\hline \multicolumn{6}{|c|}{ Tn = Perfil sob mata nativa } \\
\hline $\begin{array}{l}\mathrm{A} \\
\mathrm{AB} \\
\mathrm{BA} \\
\mathrm{Bw}_{1} \\
\mathrm{Bw}_{2}\end{array}$ & $\begin{array}{l}0,0-0,15 \\
0,15-0,35 \\
0,35-0,80 \\
0,80-1,45 \\
1,45-2,05\end{array}$ & $\begin{array}{l}420 A a \\
410 A a \\
310 A b \\
280 \\
270\end{array}$ & $\begin{array}{l}150 \mathrm{Ba} \\
150 \mathrm{Ba} \\
150 \mathrm{Ba} \\
140 \\
140\end{array}$ & $\begin{array}{l}340 \mathrm{Ab} \\
330 \mathrm{BCb} \\
420 \mathrm{Ba} \\
450 \\
460\end{array}$ & $\begin{array}{l}90 A b \\
110 A B a b \\
120 A a \\
130 \\
130\end{array}$ \\
\hline \multicolumn{6}{|c|}{ T2 = Perfil com 2 anos de cultivo } \\
\hline $\begin{array}{l}\mathrm{A} \\
\mathrm{AB} \\
\mathrm{BA} \\
\mathrm{Bw} \\
\mathrm{Bw}_{2}\end{array}$ & $\begin{array}{l}0,0-0,17 \\
0,17-0,35 \\
0,35-0,70 \\
0,70-1,40 \\
1,40-2,05\end{array}$ & $\begin{array}{l}430 A a \\
420 A a \\
310 A b \\
300 \\
300\end{array}$ & $\begin{array}{l}140 \mathrm{Ba} \\
150 \mathrm{Ba} \\
150 \mathrm{Aa} \\
140 \\
130\end{array}$ & $\begin{array}{l}330 \mathrm{ABb} \\
310 \mathrm{Cb} \\
420 \mathrm{Ba} \\
450 \\
460\end{array}$ & $\begin{array}{l}100 A a \\
120 A a \\
120 A a \\
110 \\
110\end{array}$ \\
\hline \multicolumn{6}{|c|}{ T18 = Perfil com 18 anos de cultivo } \\
\hline $\begin{array}{l}\mathrm{A} \\
\mathrm{AB} \\
\mathrm{BA} \\
\mathrm{Bw}_{1} \\
\mathrm{Bw}_{2}\end{array}$ & $\begin{array}{l}0,0-0,20 \\
0,20-0,40 \\
0,40-0,80 \\
0,80-1,40 \\
1,40-2,00\end{array}$ & $\begin{array}{l}360 \mathrm{Ba} \\
320 \mathrm{Bb} \\
260 \mathrm{BC} \\
260 \\
250\end{array}$ & $\begin{array}{l}220 A a \\
190 A b \\
150 A c \\
130 \\
150\end{array}$ & $\begin{array}{l}310 \mathrm{BCc} \\
360 \mathrm{Ab} \\
480 \mathrm{Aa} \\
500 \\
490\end{array}$ & $\begin{array}{l}110 \mathrm{Aa} \\
130 \mathrm{Aa} \\
110 \mathrm{Aa} \\
110 \\
110\end{array}$ \\
\hline \multicolumn{6}{|c|}{ T25 = Perfil com 25 anos de cultivo } \\
\hline $\begin{array}{l}\mathrm{A} \\
\mathrm{AB} \\
\mathrm{BA} \\
\mathrm{Bw}_{1} \\
\mathrm{Bw}_{2}\end{array}$ & $\begin{array}{l}0,0-0,17 \\
0,17-0,35 \\
0,35-0,70 \\
0,70-1,30 \\
1,30-2,00\end{array}$ & $\begin{array}{l}370 \mathrm{Ba} \\
350 \mathrm{Bb} \\
250 \mathrm{BC} \\
240 \\
230\end{array}$ & $\begin{array}{l}230 A a \\
210 A b \\
160 A c \\
150 \\
150\end{array}$ & $\begin{array}{l}300 C c \\
350 A b \\
490 A a \\
490 \\
510\end{array}$ & $\begin{array}{l}100 \mathrm{Aa} \\
90 \mathrm{Ba} \\
100 \mathrm{Aa} \\
120 \\
110\end{array}$ \\
\hline
\end{tabular}

Letras maiúsculas comparam tempos de cultivos diferentes na mesma profundidade. Letras minúsculas comparam profundidades dentro do mesmo tempo de cultivo. Médias seguidas de letras iguais não diferem entre si pelo teste de Tukey ao nível de 5\%.

T25 $\left(0,228 \mathrm{~m}^{3} \mathrm{~m}^{-3}\right)$ revela que o manejo do solo a longo prazo favoreceu o desenvolvimento da macroporosidade na superfície a níveis próximos do solo sob condições naturais.

Os valores de porosidade total caíram, significativamente, no primeiro plantio da cana-de-açúcar (perfil T2), nos horizontes Ap eAB. A partir daí, houve uma recuperação da por osidade total, provavel mente devida à condição semi perene e ao sistema radicular da cana-de-açúcar, chegando aos 25 anos de cultivo com uma porosidade total semel hante à do solo virgem. Os valores da macroporosidade tiveram a mesma tendência que a porosidade total, caindo, significativamente, aos dois anos de cultivo e recuperando aos 25 anos.

Apesar de o conteúdo de argila do horizonte superficial (Ap) ter diminuído com otempo de cultivo, a microporosidade aumentou nos perfis T18 e T25, provavelmente em conseqüência do aumento do conteúdo de areia fina, que compensou, em parte, a perda de argila. Verificou-se aumento significativo da microporosidade com a profundidade em todos os perfis (Quadro 2), em decorrência do aumento do teor de argila, que leva, como comenta Dixon (1991), à formação de poros pequenos e à conseqüente redução dos macroporos. Esseaumento da microporosidadeem profundidade aumenta a capacidade de retenção de água e restringe o fluxo da água nesses horizontes.

Capacidade de campo (CC), ponto de murcha permanente (PMP) eágua disponível: Observa-se, no quadro 3, que a CC e o PMP aumentaram, significativamente, após os 18 e 25 anos de cultivo, nos horizontes $A, A B$ e $B A$, em relação ao solo virgem. Também se verificam aumentos significativos com a profundidade devidos ao aumento da microporosidade e redução da macroporosidade. As variações significativas da CC e PMP com o tempo de cultivo e com a profundi dade confirmam as variações verificadas nos conteúdos de argila nas mesmas direções.

A água disponível aumentou, significativamente, nos horizontes superficiais, após 18 e 25 anos de cultivo, e com a profundidade. Tais aumentos acompanham a mesma direção dos aumentos de argila (Quadro 1) e microporosidade (Quadro 2). A única restrição ocorre no horizonte $A$, onde a água disponível aumenta com o tempo de cultivo, apesar do decréscimo de argila 
Quadro 2. Densidade do solo, porosidade total, macro e microporosidade dos horizontes coletados nos quatro perfis de latossolo amarelo

\begin{tabular}{|c|c|c|c|c|c|}
\hline \multicolumn{2}{|c|}{ Horizonte } & \multirow{2}{*}{$\begin{array}{c}\text { Densidade } \\
\text { do solo }\end{array}$} & \multicolumn{3}{|c|}{ Porosidade } \\
\hline Símbolo & Espessura & & Micro & Macro & Total \\
\hline & $\mathrm{m}$ & $\mathrm{MIg} \mathrm{m}^{-3}$ & $\ldots$ & $n^{3} m^{-3}-$ & \\
\hline \multicolumn{6}{|c|}{ Tn = Perfil sob mata nativa } \\
\hline A & $0,0-0,15$ & $1,26 \mathrm{Cc}$ & $0,261 A B c$ & $0,260 A a$ & $0,522 \mathrm{Aa}$ \\
\hline$A B$ & $0,15-0,35$ & $1,31 \mathrm{Cb}$ & $0,312 \mathrm{ABb}$ & $0,205 \mathrm{Ab}$ & $0,517 \mathrm{Aa}$ \\
\hline BA & $0,35-0,80$ & $1,42 \mathrm{Aa}$ & $0,347 \mathrm{Aa}$ & $0,123 \mathrm{AC}$ & $0,471 \mathrm{Bb}$ \\
\hline $\mathrm{Bw}_{1}$ & $0,80-1,45$ & 1,31 & & & 0,509 \\
\hline $\mathrm{Bw}_{2}$ & $1,45-2,05$ & 1,30 & & & 0,513 \\
\hline \multicolumn{6}{|c|}{ T2 = Perfil com 2 anos de cultivo } \\
\hline Ap & $0,0-0,17$ & $1,54 \mathrm{Aa}$ & $0,252 B C$ & $0,173 \mathrm{Ca}$ & $0,426 \mathrm{Cb}$ \\
\hline$A B$ & $0,17-0,35$ & $1,57 \mathrm{Aa}$ & $0,288 \mathrm{Bb}$ & $0,135 \mathrm{Bb}$ & $0,423 \mathrm{Cb}$ \\
\hline BA & $0,35-0,70$ & $1,44 \mathrm{Ab}$ & $0,338 \mathrm{Aa}$ & $0,134 \mathrm{Ab}$ & $0,473 \mathrm{Ba}$ \\
\hline $\mathrm{Bw}_{1}$ & $0,70-1,40$ & 1,36 & & & 0,491 \\
\hline $\mathrm{Bw}_{2}$ & $1,40-2,05$ & 1,18 & & & 0,569 \\
\hline \multicolumn{6}{|c|}{ T18 = Perfil com 18 anos de cultivo } \\
\hline Ap & $0,0-0,20$ & $1,39 \mathrm{Bb}$ & $0,273 \mathrm{ABC}$ & 0,197BCa & $0,470 \mathrm{Ba}$ \\
\hline$A B$ & $0,20-0,40$ & $1,49 \mathrm{Ba}$ & $0,304 \mathrm{Bb}$ & $0,142 \mathrm{Bb}$ & $0,447 \mathrm{Bb}$ \\
\hline BA & $0,40-0,80$ & $1,42 \mathrm{Ab}$ & $0,339 A a$ & $0,130 \mathrm{Ab}$ & $0,470 \mathrm{Ba}$ \\
\hline $\mathrm{Bw}_{1}$ & $0,80-1,40$ & 1,34 & & & 0,498 \\
\hline $\mathrm{Bw}_{2}$ & $1,40-2,00$ & 1,19 & & & 0,554 \\
\hline \multicolumn{6}{|c|}{ T25 = Perfil com 25 anos de cultivo } \\
\hline Ap & $0,0-0,17$ & $1,27 \mathrm{Cb}$ & $0,286 A b$ & $0,228 \mathrm{Aa}$ & $0,514 \mathrm{Aa}$ \\
\hline$A B$ & $0,17-0,35$ & $1,30 \mathrm{Cb}$ & $0,332 \mathrm{Aa}$ & $0,179 A b$ & $0,512 \mathrm{Aa}$ \\
\hline BA & $0,35-0,70$ & $1,35 \mathrm{Ba}$ & $0,345 \mathrm{Aa}$ & $0,147 \mathrm{AC}$ & $0,492 A b$ \\
\hline $\mathrm{Bw}_{1}$ & $0,70-1,30$ & 1,19 & & & 0,547 \\
\hline $\mathrm{Bw}_{2}$ & $1,30-2,00$ & 1,21 & & & 0,547 \\
\hline
\end{tabular}

Letras maiúsculas comparam tempos de cultivos diferentes na mesma profundidade. Letras minúsculas comparam profundidades dentro do mesmo tempo de cultivo. Médias seguidas de letras iguais não diferem entre si pelo teste de Tukey ao nível de 5\%.

nesse horizonte. Contudo, nesse horizonte, além da argila, a matéria orgânica exerce influência, aumentando a água disponível, como também o conteúdo de areia fina, que aumentou, significativamente, com o tempo de cultivo, o que contribuiu para compensar a perda de argila.

Levando em consideração os valores de água disponível, densidade do solo e espessura de cada horizonte, calculou-se a disponibilidade de água no primeiro 1,2 $\mathrm{m}$ de profundidade de cada perfil. Verificou-se que a lâmina d'água cresceu expressivamente, com o tempo de cultivo em relação ao solo virgem: de 8,53 cm, para o perfil Tn, e 10,21, 12,78 e 11,77 , para os perfis T2, T18 eT25, respectivamente. Há, portanto, uma alteração nas condições físicas dos solos, que se reflete por um expressivo aumento na disponi bilidade deágua para as plantas, proporcionando acréscimo de até 50\% (perfil T18) em relação ao sol o virgem. Tal mudança nas características hídricas do solo reflete as alterações na densidade, textura e porosidade dos solos.

Condutividade hidráulica saturada: 0 quadro 3 mostra a variação da condutividade hidráulica saturada, considerando o tempo de cultivo e a profundidade. Nota-se que seus valores caem, significativamente, nos horizontes AB e BA, em todos os perfis, provavelmente por causa da redução da macroporosidade e do aumento da microporosidade com a profundidade. Com relação ao tempo de cultivo, a variação nos valores da condutividade hidráulica refletem os efeitos ocorridos na densidade do solo, macro emi cropor osidade. Ocorre redução significativa aos dois anos de cultivo, refletindo queda da macroporosidade eaumento da densidade do sol o. Aos 18 e 25 anos de cultivo, os valores de condutividade hidráulica são, parcialmente, recuperados, com aumentos significativos em relação ao perfil T2, em conseqüência dos aumentos na macroporosidade e da redução na densidade do solo com maior tempo de cultivo. O maior val or observado de condutividade hidráulica ocorre no horizonteA do perfil Tn, enquanto o menor ocorre no horizonte BA do perfil T25.

Os valores da condutividade hidráulica encontrados neste trabalho são semel hantes aos obtidos por Santos (1993), em latossolos roxos, e superiores aos de Correia (1985 b), em latossolo 
Quadro 3. Valores de capacidade de campo, ponto de murcha permanente, água disponível e condutividade hidráulica saturada dos quatro perfis estudados

\begin{tabular}{|c|c|c|c|c|c|}
\hline \multicolumn{2}{|c|}{ Horizonte } & \multicolumn{2}{|c|}{ Umidade } & \multirow{2}{*}{$\begin{array}{c}\text { Água } \\
\text { disponível }\end{array}$} & \multirow{2}{*}{$\begin{array}{l}\text { Condutividade } \\
\text { hidráulica sat. }\end{array}$} \\
\hline Símbolo & Espessura & $-0,034 \mathrm{Mpa}$ & -1,52 Мра & & \\
\hline & \multirow[t]{2}{*}{$\mathrm{m}$} & \multicolumn{3}{|c|}{$\mathrm{g} \mathrm{kg}^{-1}$} & $\mathrm{~cm} \mathrm{~h}^{-1}$ \\
\hline \multicolumn{5}{|c|}{ Tn $=$ Perfil sob mata nativa } & \\
\hline A & $0,0-0,15$ & $161,7 \mathrm{Bb}$ & $123,7 \mathrm{BC}$ & $38,0 \mathrm{Bb}$ & $87,82 \mathrm{Aa}$ \\
\hline$A B$ & $0,15-0,35$ & $168,1 \mathrm{Cb}$ & $132,3 \mathrm{Cb}$ & $35,8 \mathrm{Cb}$ & $47,10 \mathrm{Ab}$ \\
\hline BA & $0,35-0,80$ & $220,1 \mathrm{Ba}$ & $171,3 \mathrm{Ba}$ & $48,8 \mathrm{Ca}$ & $6,25 \mathrm{Ac}$ \\
\hline $\mathrm{Bw}_{1}$ & $0,80-1,45$ & 232,7 & 161,2 & 71,5 & \\
\hline $\mathrm{Bw}_{2}$ & $1,45-2,05$ & 261,5 & 168,7 & 92,8 & \\
\hline \multicolumn{6}{|c|}{ T2 = Perfil com 2 anos de cultivo } \\
\hline A & $0,0-0,17$ & $132,7 \mathrm{Cc}$ & $97,2 \mathrm{Cc}$ & $35,5 \mathrm{Bc}$ & $37,22 \mathrm{Ca}$ \\
\hline$A B$ & $0,17-0,35$ & $155,5 \mathrm{Db}$ & $108,3 \mathrm{Db}$ & $47,2 \mathrm{Bb}$ & $14,24 \mathrm{Bb}$ \\
\hline BA & $0,35-0,70$ & $205,5 \mathrm{Ca}$ & $142,0 \mathrm{Ca}$ & $63,5 \mathrm{Ba}$ & $4,02 A b$ \\
\hline $\mathrm{Bw}_{1}$ & $0,70-1,40$ & 230,8 & 160,9 & 69,9 & \\
\hline $\mathrm{Bw}_{2}$ & $1,40-2,05$ & 253,6 & 166,6 & 87,0 & \\
\hline \multicolumn{6}{|c|}{ T18 = Perfil com 18 anos de cultivo } \\
\hline A & $0,0-0,20$ & $201,9 \mathrm{Ab}$ & $139,2 \mathrm{Ab}$ & $62,7 \mathrm{Ab}$ & $59,64 \mathrm{Ba}$ \\
\hline$A B$ & $0,20-0,40$ & $205,7 \mathrm{Bb}$ & $141,7 \mathrm{Bb}$ & $64,0 \mathrm{Ab}$ & $16,47 \mathrm{Bb}$ \\
\hline BA & $0,40-0,80$ & $258,7 \mathrm{Aa}$ & $172,7 \mathrm{Ba}$ & $86,0 \mathrm{Aa}$ & $5,42 A b$ \\
\hline $\mathrm{BW}_{1}$ & $0,80-1,40$ & 241,6 & 162,2 & 79,4 & \\
\hline $\mathrm{Bw}_{2}$ & $1,40-2,00$ & 273,6 & 174,6 & 99,0 & \\
\hline \multicolumn{6}{|c|}{ T25 = Perfil com 25 anos de cultivo } \\
\hline A & $0,0-0,17$ & $208,8 \mathrm{AC}$ & $141,7 \mathrm{AC}$ & $67,1 \mathrm{Ab}$ & $58,45 \mathrm{Ba}$ \\
\hline$A B$ & $0,17-0,35$ & $218,7 A b$ & $147,9 \mathrm{Ab}$ & $70,8 \mathrm{Ab}$ & $26,33 \mathrm{ABb}$ \\
\hline BA & $0,35-0,70$ & $267,1 \mathrm{Aa}$ & $183,4 \mathrm{Aa}$ & $83,7 \mathrm{Aa}$ & $2,58 \mathrm{Ac}$ \\
\hline $\mathrm{Bw}_{1}$ & $0,70-1,30$ & 249,4 & 170,0 & 79,4 & \\
\hline $\mathrm{Bw}_{2}$ & $1,30-2,00$ & 272,9 & 177,4 & 95,5 & \\
\hline
\end{tabular}

Letras maiúsculas comparam tempos de cultivos diferentes na mesma profundidade. Letras minúsculas comparam profundidades dentro do mesmo tempo de cultivo. Médias seguidas de letras iguais não diferem entre si pelo teste de Tukey ao nível de 5\%.

amarelo muito argiloso, refletindo os valores da macroporosidade que são também mais el evados. Cadima Z. (1984), trabalhando com um oxisol muito argil oso detabuleiro (Haplorthox), encontrou val ores de condutividade hidráulica saturada bem mais baixos, fato, provavelmente, atribuído à menor porosidade total e à maior densidade do solo em relação ao solo deste trabal ho.

\section{CONCLUSÕES}

1. O aumento significativo da relação textural deveu-se à redução do conteúdo de argila nos horizontes superficiais e ao aumento significativo nos horizontes subsuperficiais dos sol os cultivados.

2. O manejo adotado promoveu novo equilíbrio nas propriedades físicas, com uma recuperação parcial da estrutura, aumento da porosidade total, macroporosidade e redução da densidade do solo dos horizontes compactados (Ap e AB). Observou-se, também, sensível aumento da disponibilidade deágua nesses horizontes superficiais, após 18 anos de cultivo.
3. A coesão do horizonte BA é provocada por um adensamento de origem genética, pois ocorre com a mesma intensidade no perfil sob mata nativa.

4. A redução da condutividadehidráulica saturada nos sol os cultivados em rel ação ao sol o sob mata nativa não aumentou com os anos de cultivo.

\section{LITERATURA CITADA}

CADIMA Z., A. Condutividade hidráulica de um Oxisol (Haplorthox) variação tabuleiro. R. Theobroma, Ilhéus, 14:149-157, 1984.

CAMARGO, O. A. Efeitos da compactação em características do solo. In: Compactação do solo e desenvol vimento de plantas. Campinas, Fundação Cargill, 1983. 44p.

CERRI, C.C.; FELLER, C. \& CHAUVEL,A. Evolução das principais propriedades de um latossolo vermelho escuro após desmatamento e cultivo por doze e cinquenta anos com canade-açucar. Cahiers Orstom, sér. Pédologie, Bondy, 26:37-50, 1991. 
CORREIA, J.C. Efeito de métodos de cultivo em algumas propriedades físicas de um Latossol o Amarelo muito argiloso do Estado do Amazonas. Pesq. agrop. bras., Brasília, 20:13171322, 1985a.

CORREIA, J.C. Características físicas de um Latossolo Amarelo muito argiloso (TypicAcrorthox) do Estado doAmazonas, sob diferentes métodos de preparo do solo. Pesq. agrop. bras., Brasília, 20:1381-1387, 1985b.

CORSINI, P.C. Problemas causados pela compactação dos solos. STAB: açúcar, ál cool e subprodutos, Piracicaba, 11:8-12, 1993.

DIXON, J.B. Roles of clays in soils. App. Clay Sci. Amsterdam, 5:489-503, 1991.

EMBRAPA. Manual de métodos de análises do sol os. Rio deJ aneiro, SNCLS, 1979. 247p.

FARIAS, G.S.; CASSOL, E.A. \& MIELNICZUK, J. Efeito de sistemas de cultivo sobre a porosidade e retenção da água em um solo laterítico bruno-avermelhado distrófico (paleudult). Pesq. agrop. bras., Brasília, 20:1389-1393, 1985.

GOVES, G.; VANDAELE, K.; DESMET, P.; CAVALCANTI, A.C.; PESSOA, S.C.P. \& SILVEIRA, C.O. The role of tillage in soil redistribution on hillslopes. Europ. J. Soil Sci., Oxford, 45:469478, 1994.

GROHMANN, F. Distribuição e tamanho de poros em três tipos de solos do Estado de São Paulo. Bragantia, Campinas, 19:319329, 1960.

J ACOMINE, P.K.T.; POESEN, J \& \& BUNTE, K. Levantamento Exploratório Reconhecimento de Solos do Estado deAlagoas. Recife, EMBRAPA, Centro de Pesquisas Pedológicas. SUDENE-DRN, 1975. 531p. (Boletim Técnico, 35)

KLUTE, A. \& DIRKSEN, C. Hydraulic conductivity and difusivity laboratory methods. In: KLUTE, A., ed. Methods of soil analysis; physical and mineralogical methods. Madison, American Society of Agronomy, Part I, 1986.

LEMOS, R.C. \& SANTOS, R.D. dos. Manual de descrição e col eta de solo no campo. Campinas, SBCS/SNLCS, 1984. 45p.

LIMA, L.da R.F. Determinação da condutividade hidráulica saturada em solos aluvionais por método de campo e laboratório. Recife, Universidade Federal Rural de Pernambuco, 1986. 81p. (Dissertação de Mestrado)

MCAFEE, M.; LINDSTRÖM, J. \& J OHANSSON, W. Aeration changes ofter irrigation in a clay soil. J. Soil Sci., Oxford, 40:718-729, 1989.
MEEK, B.D.; RECHEL, E.R.; CARTER, L.M. \& DeTAR, W.R. Bulk density of a Sand Loam: traffic, tillage and irrigation - method effects. Soil Sci. Soc. Am. J., Madison, 56:562-565, 1992.

PANOSO, A.L. Latossolo Vermel ho Amarelo de tabuleiros do Espírito Santo: formação, características e classificação. Recife, Escola Superior de Agricultura da Universidade Federal Rural de Pernambuco, 1976. 116p. (Tese de Livre Docente)

PEREZ FILHO, A.; TESTEZLAF, R. \& TERESO, M.J . de A. Efeito da irrigação na compactação de latossolos argilosos submetidos ao uso agrícola intenso. Eng. Agrí., Campinas, 13:39-55, 1993.

ORLANDO FILHO, J.; BITTENCOURT, V.C. de. \& ALVES, M.C. A plicação de vinhaça em solo arenoso do Brasil e poluição do lençol freático com nitrogênio. STAB: açúcar, álcool e subprodutos, Piracicaba, 13:14-16, 1995.

RICHARDS, L.A. Diagnosis and Improvement of saline and alkaline soils. Washington, USDA, U.S. Government Printing Office, 1954, 160 p. (Agriculture Handbook, 60)

ROMERO de CARVALHO, S.; BRUAND, A.; HARDY, M.; LEPRUM, J.C. \& JAMAGNE, M. Tassement des sols ferrallitiques Podzólico Vermelho Amarelo sous culture de canne à sucre (état de Rio de J aneiro, Brézil): apport d'une analyse de la porosité associée a une connaissance détaillée de la phase minérale. Cahiers Orstom, sér. Pedologie, Bondy. 26:195-212, 1991.

SANTOS, J .C.F. Comportamento de propriedades físicas e químicas de dois latossolos roxos sob diferentes sistemas de rotação de culturas em plantio direto. Lavras, Escola Superior de Agricultura de Lavras, 1993, 101p. (Dissertação de Mestrado)

SILVA, M.S.L. da. \& RIBEIRO, M.R. Influência do cultivo contínuo da cana-de-açucar em propriedades morfológicas e físicas de solos argilosos de tabuleiros do Estado de Alagoas. R. bras. Ci. Solo, Campinas, 16:397-402, 1992.

SILVA, J.A.A. \& SILVA, I.P. da. Estatística experimental aplicada à ciência florestal. Recife, Imprensa Universitária, UFRPE, 1982. $294 \mathrm{p}$

VOMOCIL, J.A. Porosity. In: BLACK, C.A. ed. Methods of soil analysis; physical and mineralogical properties, includind statistics of measurement and sampling. Madison, America Society Agronomy, part I, 1965. 191p.

ZONTA, E.P. \& MACHADO, A.A. SANEST - Sistema de Análise Estatística. São Paulo, Escola Superior de Agricultura Luiz de Queiroz (ESALQ)-USP. Departamento de Matemática e Estatística, 1980. 